\title{
Improvement of colchicine oral bioavailability by incorporating eugenol in the nanoemulsion as an oil excipient and enhancer
}

This article was published in the following Dove Press journal:

International Journal of Nanomedicine

21 June 201 I

Number of times this article has been viewed

\section{Qi Shen \\ Ying Wang \\ Yi Zhang}

School of Pharmacy, Shanghai Jiao Tong University, Dongchuan Road 800, Shanghai 200240,

People's Republic of China
Correspondence: Qi Shen School of Pharmacy, Shanghai Jiao

Tong University, Dongchuan Road 800, Shanghai 200240,

People's Republic of China

Tel +8602 I 34204049

Fax +86 02I 34204049

Email qshen@sjtu.edu.cn

\begin{abstract}
The effect of eugenol on colchicine transport across an isolated rat intestinal membrane was studied using an in vitro diffusion chamber system. We found that eugenol increased the absorptive transport of the drug efficiently. The effect of eugenol on intestinal absorption of colchicine in an oral administrative nanoemulsion formulation was also demonstrated in vivo. The colchicine nanoemulsion was prepared with isopropyl myristate, eugenol, Tween80, ethanol and water, and eugenol was used as an oil phase in the formulation; an average particle size of this nanoemulsion was $41.2 \pm 7.2 \mathrm{~nm}$. The permeation of colchicine in the nanoemulsion across the intestinal membrane was significantly different from that of the control group ( $0.2 \mathrm{mM}$ colchicine). Finally, co-administration of eugenol in colchicine nanoemulsion to enhance the colchicine bioavailability was investigated by an oral administration method. After oral administration of colchicine $(8 \mathrm{mg} / \mathrm{kg})$ in the form of either the nanoemulsion or in free colchicine solution, the relative bioavailability of nanoemulsion and eugenol-nanoemulsion were enhanced by about 1.6- and 2.1-fold, respectively, compared with free colchicine solution. The procedure indicated that the intestinal absorption of colchicine was enhanced significantly by eugenol in the tested nanoemulsion. All the results suggested that eugenol is an efficient component in an oral administrative formulation for improving the intestinal absorption of colchicine.
\end{abstract}

Keywords: intestinal absorption, nanoemulsion, absorption enhancement, eugenol

\section{Introduction}

Oral administration of drugs is a preferred route by virtue of convenience and compliance. However, the delivery of some drugs by the oral route is considered to be a challenge due to the poor water solubility, poor permeability, and rapid metabolism. To achieve oral delivery of drugs with poor solubility and poor permeability properties, many strategies have been developed to improve bioavailability, such as using absorption enhancers, and developing new pharmaceutical excipients ${ }^{1-3}$ and novel dosage forms, such as nanoemulsion. ${ }^{4,5}$ Several pharmaceutical excipients have been shown to reduce the efflux function of permeability glycoprotein (P-gp) in the intestine, and hence increase the absorption of drugs which act as P-gp substrates. ${ }^{6-10}$

Some terpenoids were recently reported as P-gp substrates or inhibitors, ${ }^{11-13}$ and Yoshida et al found that natural products containing terpenoids may inhibit P-gp-mediated transport. ${ }^{14,15}$ Eugenol is a simple monocyclic terpene, which has been reported as a penetration enhancer in the transdermal drug delivery system. ${ }^{16,17}$ Furthermore, eugenol could increase the sensitivity of Hep G2 cells to anti-cancer drugs, accompanied by a reduction in the expression of multidrug resistance protein 2 (MRP2). ${ }^{18}$ There are few 
reports, however, on the use of eugenol in oral administration formulations to enhance bioavailability of poor permeability drugs.

P-glycoprotein can transport colchicine and expel it from the cell, preventing intracellular penetration and causing resistance to treatment. The key colchicine targets may explain the wide interindividual variations in responses to the drug. When inhibitors or modulators are prescribed in combination, changes in P-glycoprotein activity may lead to intracellular accumulation of colchicine. ${ }^{19}$

In this paper, the effects of eugenol on the intestinal transportation and absorption of colchicine have been demonstrated in vitro. An oral administrative nanoemulsion of colchicine was also prepared using eugenol as the oil phase. The effect of eugenol in this nanoemulsion formulation was investigated in vitro and in vivo, and the improvement of colchicine bioavailability by addition of eugenol was confirmed.

\section{Materials and methods Materials}

Colchicine was purchased from Shanghai Chemical Reagents Institute (Shanghai, China). Eugenol was bought from Feixiang Chemical Industries, Ltd (Shanghai, China). All other reagents were of analytical grade.

\section{Preparation of drug solution}

Colchicine was dissolved in $\mathrm{pH} 7.4$ Tris-HEPES buffer solution to yield a final concentration, $0.2 \mathrm{mM}$ colchicine was used for the in vitro transport study, while $8 \mathrm{mg} / \mathrm{kg}$ of colchicine was used for an in vivo absorption study. In some experiments, 0.5 to $2 \%(\mathrm{w} / \mathrm{w})$ of eugenol was added to the donor solution.

\section{Nanoemulsion preparation}

A pseudo-ternary phase diagram was constructed to identify the formation of nanoemulsion. The nanoemulsion of colchicine was prepared by solubilization of colchicine in ethanol, which was used as co-surfactant. The oil phase (isopropyl myristate), eugenol, and surfactant (Tween80) were added slowly under gentle stirring until a homogeneous mixture formed. Then water was added dropwise at $37^{\circ} \mathrm{C}$ under stirring at $600 \mathrm{rpm}$.

\section{Nanoemulsion droplet size analysis and morphology observation by transmission electron microscope (TEM)}

The particle size of the nanoemulsion was determined by a photo correlation spectroscopy instrument (Malvern Instruments, Malvern, UK) at $25^{\circ} \mathrm{C}$. The morphology of the droplet was observed using transmission electron microscopy (JEM-2010; JEOL, Tokyo Japan). One drop of diluted samples was deposited on a film-coated 200-mesh copper specimen grid and allowed to stand for 10 minutes after which any excess fluid was removed with filter paper. The grid was then stained with one drop of $3 \%$ phosphotungstic acid and allowed to dry for 5 minutes before examination with an electron microscope.

\section{In vitro transport of colchicine}

The transport of colchicine across a rat intestinal membrane was studied with the diffusion chamber. ${ }^{20,21}$ Male SpragueDawley rats, weighing 250 to $300 \mathrm{~g}$, were fasted overnight. The experimental procedures in this article were in accordance with the guidelines of the Animal Ethics Committee at Shanghai Jiao Tong University.

The intestine was exposed through a midline abdominal incision, removed, and washed in ice-cold saline. Intestinal segments, excluding Peyer's patches, were isolated and immersed in ice-cold Tris-HEPES buffer solution ( $\mathrm{pH}$ 7.4) containing $25 \mathrm{mM}$ HEPES, $140 \mathrm{mM} \mathrm{NaCl}, 5.4 \mathrm{mM} \mathrm{KCl}, 1.8 \mathrm{mM} \mathrm{CaCl}_{2}$, $0.8 \mathrm{mM} \mathrm{MgSO}, 5 \mathrm{mM}$ glucose, and $1 \mathrm{M}$ Tris solution. Segments were cut open and the intestinal sheets were mounted onto the pins of the cell, and the half-cells were clamped together. Drug solution $(7 \mathrm{~mL}$ ) of $0.2 \mathrm{mM}$ colchicine (control), or $0.2 \mathrm{mM}$ colchicine with eugenol, nanoemulsion, and eugenol-nanoemulsion, was added to the donor site, respectively, whereas the same volume of drug-free buffer was added to the opposite side. The temperature of intestinal membranes was maintained at $37^{\circ} \mathrm{C}$, and both fluids were circulated by gas lift with $95 \% \mathrm{O}_{2} / 5 \% \mathrm{CO}_{2}$. During the transport studies, aliquots were taken from the receptor chamber at predetermined times up to 2 hours. The receptor chamber samples were replaced with an equal volume of appropriate buffer. The permeated drugs were assayed using HPLC. The apparent permeability coefficients $\left(P_{\text {app }}\right)$ of drugs were calculated from the slope of the linear portion of permeability-time profiles of drugs by the relationship: $P_{\text {app }}=\left(\mathrm{d} X_{\mathrm{R}} / \mathrm{d} t\right) \times\left(1 / A \cdot \mathrm{C}_{0}\right) \mathrm{p} 1 / 60$, where $P_{\text {app }}$ is the apparent permeability coefficient, $X_{\mathrm{R}}$ is the amount of drug in the receptor side, $A$ is the diffusion area, and $\mathrm{C}_{0}$ is the initial concentration of drug in the donor side.

The absorption enhancement ratio $(\mathrm{R})$ was calculated from $P_{\text {app }}$ values from the mucosal to the serosal side as follows:

$$
\mathrm{R}=P_{\text {app }}(\text { sample }) / P_{\text {app }}(\text { control })
$$

The efflux ratio was used to evaluate the extent of efflux, ${ }^{22-25}$ which was defined as follows: 


$$
\text { Efflux ratio }=P_{\text {app }} \mathrm{sm} / P_{\text {app }} \mathrm{ms}
$$

where $P_{\text {app }} \mathrm{sm}$ is the average of the permeability coefficient from the serosal to the mucosal side and $P_{\text {app }} \mathrm{ms}$ is the average of the permeability coefficient from the mucosal to the serosal side.

In order to confirm whether the viability of the intestinal membrane was maintained during the transport studies, the transport of trypan blue dye was examined. $1 \%$ of trypan blue solution was added to the mucosal side and the transport of this dye across the rat intestinal membrane was monitored for 2 hours by an in vitro diffusion chamber method. There was no transport of the dye, confirming that the viability of the intestinal membranes was maintained during the transport experiments.

\section{Pharmacokinetic study}

Male Sprague-Dawley rats were fasted overnight with free access to water before drug administration. The experiments were carried out in accordance with the guidelines of Animal Ethics Committee at Shanghai Jiao Tong University. Free colchicine solution as a control sample, the nanoemulsion formulation (present and absent eugenol), and the control sample were administered orally ( $8 \mathrm{mg} / \mathrm{kg}$ of colchicine) with a blunt needle via the esophagus into the stomach. Subsequently, blood samples $(200 \mu \mathrm{L})$ were collected into heparinized microcentrifuge tubes from the caudal vein at a designated time. Blood samples were centrifuged for 5 minutes at $5000 \times g$ and the concentrations of colchicine in the plasma were determined by HPLC (see Analysis of drug concentration). The area under the plasma concentration time curve (AUC) from time zero to final sampling time ( 8 hours) was calculated by the linear trapezoidal rule. The peak plasma concentration $\left(\mathrm{C}_{\max }\right)$ and the time to reach the peak plasma concentration $\left(\mathrm{T}_{\max }\right)$ were observed values from the experimental data.

\section{Analysis of drug concentration}

Samples obtained from in vivo absorption studies were assayed on a reversed-phase HPLC system containing $5 \mu \mathrm{m}$ Cosmosil $(4.6 \mathrm{~mm} \times 15 \mathrm{~cm})$ particles in an analytical column from Dikama Technologies, a Shimadzu LC-20A pump system, a Shimadzu LC-20A autoinjector, and a SPD-20A UV-VIS detector. The mobile phase for colchicine analysis was $0.05 \mathrm{~mol} / \mathrm{L}$ potassium dihydrogen phosphate solution ( $\mathrm{pH}$ 5.5):methanol = 55:45. The flow rate was $1.0 \mathrm{~mL} \mathrm{minute}^{-1}$. The concentrations of colchicine were determined using a UV detector (Shimadzu LC-20A) at $350 \mathrm{~nm}$ wavelengths.

When the concentrations of colchicine in plasma were determined, $400 \mu \mathrm{L}$ acetonitrile were added to $0.1 \mathrm{~mL}$ of the plasma samples. These were then mixed using a vortex mixer and centrifuged at $3000 \mathrm{rpm}$ for 5 minutes. The supernatant was transferred to a clean test tube and evaporated in a centrifugal evaporator at $40^{\circ} \mathrm{C}$, the residue was then dissolved in a mobile phase solution, and the solution $(20 \mu \mathrm{L})$ was injected into the HPLC for analysis.

\section{Statistics}

Results are expressed as the mean of SD of at least three experiments. Statistical significance was assessed using Student's $t$-test or Dunnett's test for multiple comparisons with $P<0.05$ as the minimal level of significance.

\section{Results \\ Characteristics and physicochemical properties of colchicine nanoemulsion}

The ternary phase diagrams of a system containing isopropyl myristate, eugenol, Tween80, ethanol, and distilled water are shown in Figure 1. A transparent nanoemulsion existed in the area. An optimal nanoemulsion was composed of 5\% isopropyl myristate/eugenol (w/w, 3/2), 30\% Tween 80 and $10 \%$ ethanol (surfactant/cosurfactant ratios, $\mathrm{k}_{\mathrm{m}}=3$ ), and $55 \%$ of aqueous solution $(\mathrm{w} / \mathrm{w})$. The nanoemulsion was stable at room temperature and phase separation did not occur.

Particle size of nanoemulsion was one of the most important properties. As a result, the average droplet size of the colchicine nanoemulsion was $41.2 \mathrm{~nm} \pm 7.2$. The morphology of colchicine nanoemulsion was characterized using TEM (Figure 2). It showed a spherical shape and uniform droplet size of nanoemulsion. Samples were diluted with distilled water before testing to avoid multiscattering. After that, the droplet size of the diluted nanoemulsion was

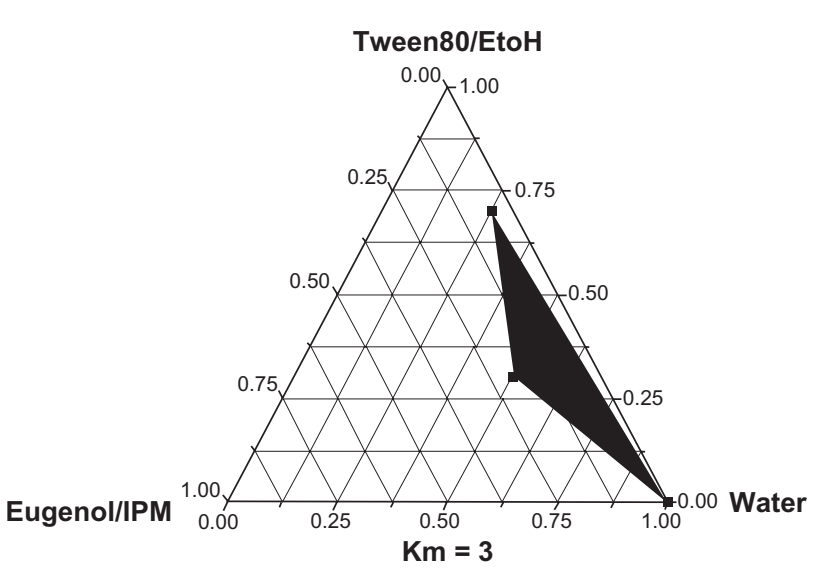

Figure I The ternary phase diagram of the colchicine nanoemulsion system containing Tween80, ethanol, distilled water and isopropyl myristate (IPM), eugenol as surfactant, water, and oil system. The shaded areas in the phase diagram represent clear and transparent nanoemulsions. 


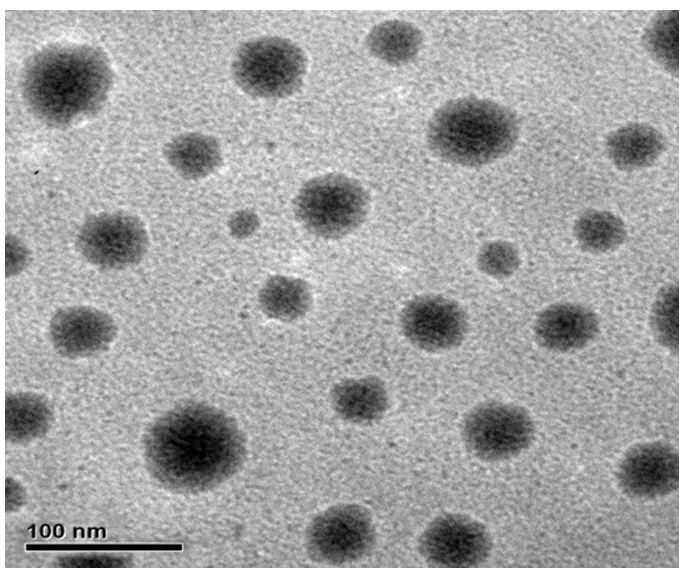

Figure 2 Transmission electron microphotograph of colchicine nanoemulsion.

not significantly changed. The mean particle size remained consistent up to 3 months at room temperature.

\section{Effects of various concentrations of eugenol on the permeability of colchicine across the intestinal membranes}

Figure 3 shows the effects of various concentrations of eugenol on the absorptive transport of colchicine in the rat jejunal membranes. The absorptive transport of colchicine was gradually increased as the concentration of eugenol increased, and the absorptive transport of colchicine was enhanced by the addition of eugenol at concentrations over the range of $0.5 \%$ to $2 \%(\mathrm{w} / \mathrm{w})$.

\section{Effects of nanoemulsions incorporating eugenol on the permeability of colchicine across the intestinal membranes}

Figure 4 shows the effect of eugenol incorporated nanoemulsion on the absorptive and secretory transport of colchicine in the rat jejunal membranes.

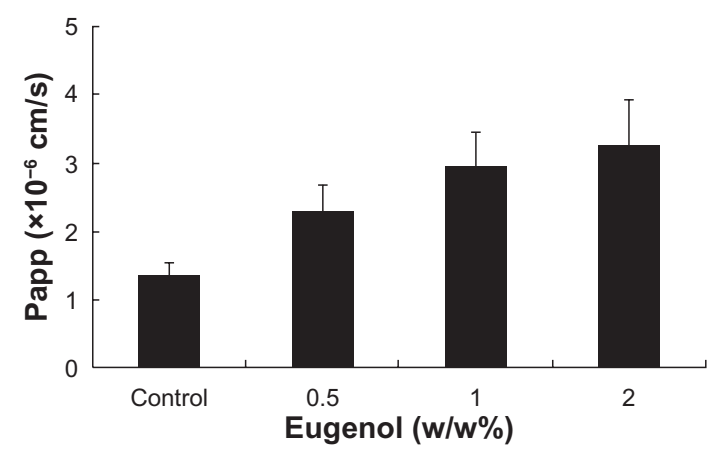

Figure 3 Concentration-dependent effects of eugenol on absorptive (mucosal to serosal, $M$ to S) transport of colchicine in the rat jejunum. The concentrations of eugenol were $0.5 \%-2 \% \mathrm{w} / \mathrm{w}$. Results are expressed as the mean \pm SD of at least 3 experiments.
The absorptive transport of colchicine was enhanced by the nanoemulsion and eugenol incorporated nanoemulsion, which from each formulation was in the following order: eugenol nanoemulsion $>$ eugenol $>$ nanoemulsion without eugenol $>$ control group (colchicine solution). The secretory transport of colchicine nanoemulsion decreased compared with the control. The order was: eugenol nanoemulsion $>$ eugenol $>$ nanoemulsion without eugenol $>$ control group (colchicine solution).

The Papp values of colchicine from mucosal to serosal and serosal to mucosal efflux were compared and results are shown in Table 1 . The results show that the permeability coefficient of the eugenol, nanoemulsion, and eugenolnanoemulsion was significantly higher than that of the control in the mucosal to serosal transport. In particular, the Papp values of colchicine from eugenol-nanoemulsion was around 2.76 times higher than that from the control group.

Table 1 also shows that the serosal to mucosal transport, the permeability coefficient of eugenol, and eugenolnanoemulsion significantly decreased compared with the control. For the control, the serosal to mucosal permeability coefficient was higher than that of mucosal to serosal value, indicating that colchicine is a substrate of P-gp.

\section{Effect of nanoemulsions incorporating eugenol on the intestinal absorption of colchicine by an in vivo oral administration method}

Finally, the effect of eugenol on the intestinal absorption of colchicine was examined by in vivo absorption studies. Therefore, a solution containing colchicine and colchicine nanoemulsion which incorporated eugenol were administered orally, respectively, with a blunt needle via the esophagus into stomach.

Figure 5 shows plasma concentration-time profiles of colchicine after the oral administration of colchicine solution and colchicine nanoemulsion. A significant increase in the AUC of colchicine was observed when the colchicine nanoemulsion was orally administered. Table 2 shows the pharmacokinetic parameters $\left(\mathrm{C}_{\max }, \mathrm{T}_{\max }\right.$, and $\left.\mathrm{AUC}_{0-8 \mathrm{~h}}\right)$ of colchicine after its oral administration. We observed a significant increase in $\mathrm{C}_{\text {max }}$ and $\mathrm{AUC}_{0-8 \mathrm{~h}}$ values after oral administration of colchicine in eugenol nanoemulsion. These findings suggest that eugenol might be a useful excipient for improving the intestinal absorption of colchicine.

\section{Discussion}

The present study demonstrated that eugenol could increase the absorptive transport of colchicines, and could 

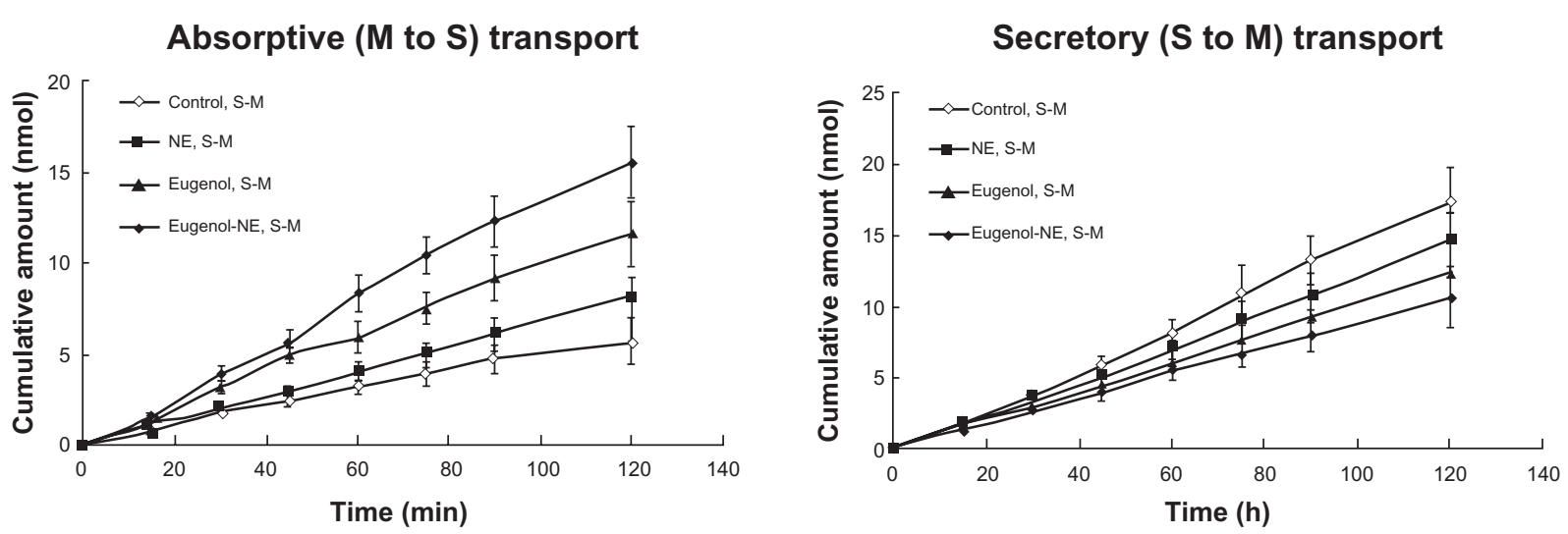

Figure 4 Time course of absorptive (mucosal to serosal, $M$ to $S$ ) and secretory ( $S$ to $M$ ) transport of colchicine across the rat jejunal membrane in the presence of $2 \%$ eugenol $(\mathrm{w} / \mathrm{w})$, nanoemulsion (NE) and eugenol-nanoemulsion (eugenol-NE). Results are expressed as the mean \pm SD of at least three experiments.

decrease the secretory transport in the intestine. The efflux ratio of colchicine was apparently reduced in the presence of eugenol compared with the control. Previous studies indicated that the secretory direction transport of colchicine was inhibited in the presence of verapamil in intestinal membrane and Caco- 2 cell. ${ }^{26}$ In addition, Dahan and Amidon reported that the secretion of colchicine was significantly inhibited and its absorption was significantly enhanced by several P-gp substrates including verapamil and quinidine in Caco- 2 cell.

In the present study, we found that eugenol could increase the absorptive transport of colchicine. However, the mechanism in the intestine was not fully studied in this paper. Eugenol can be used as percutaneous absorption enhancer, as a result of lipid extraction and improvement in the partitioning of the drug to the stratum corneum. ${ }^{16}$ Yoshida et al reported that some natural products containing terpenoids may inhibit P-gp-mediated transport and interact with P-gp substrates in the intestinal absorption process, and might decrease the expression of P-gp. ${ }^{15}$

Eugenol is not completely soluble in water, and was an oil excipient and enhancer in the nanoemulsion. Nanoemulsion systems are mixtures of oils, surfactants, co-surfactant, and water, which can produce nanoemulsions with a particle size $<100 \mathrm{~nm}$, and are clear and transparent.
Thus nanoemulsion systems can help solubilize eugenol and colchicine.

It was demonstrated that multidrug resistance protein 2 (MRP2) is also involved in the efflux process of colchicine ${ }^{27}$ and that eugenol could reduce the expression of MRP2. ${ }^{18}$ Therefore eugenol may play a potential role in the effects of MRP2 on the efflux of colchicine.

Another possibility to way of increasing the transport of colchicine across the intestinal membrane in the presence of eugenol is that this excipient might loosen the tight junction of the epithelium, thereby increasing the transport of drugs via a paracellular pathway. Chitosan-4 thiobutylamidine/ glutathione (Ch-TBA/GSH) can significantly increase the intestinal absorption and oral bioavailability of rhodamine123, a P-gp substrate, of which a possible reason is opening the tight junctions. ${ }^{6}$

Investigating the intestinal absorption is important, not only by in vitro studies but also by in vivo studies, which evaluate how pharmaceutical excipients improve the intestinal absorption of P-gp substrates. Bittner et al reported that a commonly used surfactant, vitamin E-TPGS, and solutol HS15 could improve the intestinal absorption of colchicine after oral administration in rats. ${ }^{28}$ In the present study, therefore, colchicine was selected as a model of P-gp substrate and we examined intestinal absorption

Table I Effects of eugenol and nanoemulsion (NE) on the permeability of colchicine across the intestinal jejunal membranes

\begin{tabular}{lllll}
\hline Excipient & $\mathbf{P}_{\text {app }}\left(\times 1 \mathbf{0}^{-6} \mathbf{c m} / \mathbf{s}\right)$ & & & $\begin{array}{l}\text { Absorption } \\
\text { enhancement ratio: } \mathbf{R}\end{array}$ \\
\cline { 2 - 3 } & $\mathbf{M}$ to $\mathbf{S}$ & $\mathbf{S}$ to $\mathbf{M}$ & & Efflux ratio \\
\hline Control & $1.34 \pm 0.28$ & $4.81 \pm 0.26$ & 2.33 & 3.59 \\
$2 \%$ eugenol & $3.13 \pm 0.15^{*}$ & $3.07 \pm 0.12^{* *}$ & 1.58 & 0.98 \\
Nanoemulsion & $2.12 \pm 0.32^{*}$ & $3.43 \pm 0.47^{*}$ & 2.76 & 1.62 \\
Eugenol-NE & $3.70 \pm 0.37^{* *}$ & $2.86 \pm 0.19^{* *}$ & 0.77
\end{tabular}

Notes: Each value represents the mean \pm SD of at least three experiments; $* P<0.05$; $* * P<0.01$, compared with control. 


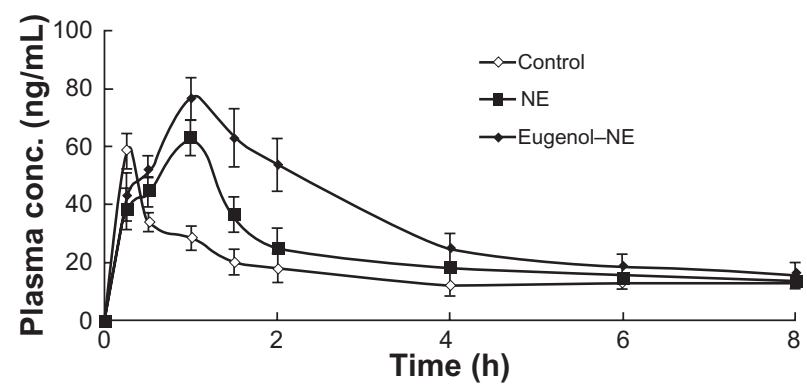

Figure 5 Plasma concentration-time profile of colchicine after oral administration with nanoemulsion (NE) or with eugenol nanoemulsion (eugenol-NE). Results are expressed as the mean \pm SD of at least 3 experiments.

of colchicine in the presence or absence of eugenol by the in vivo method.

As shown in Figure 5, we found a significant increase in $\mathrm{C}_{\max }$ and $\mathrm{AUC}_{0-8 \mathrm{~h}}$ values after administration of colchicine with eugenol incorporated in nanoemulsion. We previously demonstrated that PEG20,000 could increase the intestinal absorption of rhodamine 123 by the in situ closed-loop method. ${ }^{9}$ Therefore, it can be suggested that eugenol might have a potential role in intestinal P-gp, and could increase the absorption of colchicine from the small intestine, although we have no direct evidence to support these mechanisms at present. $\mathrm{T}_{\max }$ was obtained from the experimental measurements; the eugenol-NE $\mathrm{T}_{\text {max }}$ of colchicine occurs later than that of the control, the reason for which can be speculated as the process of drug release from the nanoemulsion. According to the references, many formulations have similar reactions. $^{29-31}$

Nanoemulsion is a lipid-based delivery system, and has some advantages including high solubilization, thermodynamic stability, and surfactant-induced permeability enhancement..$^{32,33}$ The nanoemulsion system is considered to be an alternative for the oral delivery of some drugs, such as nitrendipine, ibuprofen, and docetaxel. Therefore employing the nanoemulsion formulation can improve their oral bioavailability. ${ }^{4,5,34}$

A number of studies have been conducted to increase the poor absorption of some drugs using the co-administration

Table 2 Pharmacokinetic parameters of colchicine after oral administration in rats in the presence or absence of eugenolnanoemulsion (eugenol-NE)

\begin{tabular}{llcl}
\hline & $\mathbf{C}_{\max }(\mathbf{n g} / \mathbf{m L})$ & $\mathbf{T}_{\max }(\mathbf{h})$ & AUC $_{0 \rightarrow 8}(\mathbf{n g} / \mathbf{m L} \cdot \mathbf{h})$ \\
\hline Control & $58.34 \pm 6.12$ & $0.25 \pm 0.03$ & $129 \pm 20$ \\
$\mathrm{NE}$ & $62.83 \pm 9.32$ & $1.0 \pm 0.24^{*}$ & $212 \pm 39^{*}$ \\
Eugenol-NE & $76.45 \pm 8.3^{*}$ & $1.0 \pm 0.31^{*}$ & $271 \pm 36^{* *}$ \\
\hline
\end{tabular}

Notes: Each value represents the mean $\pm S D$ of at least three experiments; $* P<0.05 ; * * P<0.0$ I, compared with the control. of enhancers. Hirunpanich's studies indicated that ethyl docosahexaenoate, which was incorporated into a nanoemulsion formulation as an oil ingredient, ${ }^{35}$ can improve the oral bioavailability of cyclosporine A. Our study demonstrated that eugenol, incorporated into a nanoemulsion, could also enhance the permeation and oral bioavailability of colchicine. Therefore, based on these findings, our present study was well correlated with studies by Hirunpanich and Sato. ${ }^{35}$

Although studies have investigated how the eugenolnanoemulsion drug delivery system can enhance permeation and oral bioavailability, the therapeutic potential of this delivery system and the possibilities of applying eugenol as a pharmaceutical excipient need further research in the future.

\section{Conclusion}

In conclusion, eugenol could improve the intestinal absorption of drugs. The present investigation also developed a novel nanoemulsion formulation of eugenol as an oil ingredient and demonstrated that eugenol-nanoemulsion exhibited a higher bioavailability of colchicine than the control group. Thus, eugenol in oral drug formulations might be useful for improving the intestinal absorption of P-gp substrates including colchicine or as an excipient of nanoemulsions.

\section{Acknowledgments}

The work was supported by the Shanghai Municipal Committee of Science and Technology (Grant No. 08DZ1971304) and the National Basic Research Program of China (973 Program), No. 2007CB936004.

\section{Disclosure}

The authors declare no conflicts of interest in this work.

\section{References}

1. Lee VHL, Yamamoto A. Penetration and enzymatic barriers to peptide and protein absorption. Adv Drug Deliv Rev. 1989;4:171-207.

2. Aungst BJ. Intestinal permeation enhancers. J Pharm Sci. 2000;89: 429-442.

3. Li X, Shen Q. Determination of Daidzein in Rat Plasma by LC. Chromatographia. 2008;68:201-205.

4. Araya $\mathrm{H}$, Tomita M, Hayashi M. The novel formulation design of $\mathrm{O} / \mathrm{W}$ microemulsion for improving the gastrointestinal absorption of poorly water soluble compounds. Int J Pharm. 2005;305:61-74.

5. Yin YM, Cui FD, Mu CF, et al. Docetaxel microemulsion for enhanced oral bioavailability: preparation and in vitro and in vivo evaluation. J Control Release. 2009;140:86-94.

6. Föger F, Schmitz T, Bernkop-Schnürch A. In vivo evaluation of an oral delivery system for P-gp substrates based on thiolated chitosan. Biomaterials. 2006;27:4250-4255. 
7. Johnson BM, Charman WN, Porter CJ. An in vitro examination of the impact of polyethylene glycol 400, Pluronic P85, and vitamin E d-alphatocopheryl polyethylene glycol 1000 succinate on P-glycoprotein efflux and enterocyte-based metabolism in excised rat intestine. AAPS Pharm Sci. 2002;4:1-13.

8. Cornaire G, Woodley J, Hermann P, Cloarec A, Arellano C, Houin G. Impact of excipients on the absorption of P-glycoprotein substrates in vitro and in vivo. Int J Pharm. 2004;278:119-131.

9. Shen Q, Lin Y, Handa T, et al. Modulation of intestinal P-glycoprotein function by polyethylene glycols and their derivatives by in vitro transport and in situ absorption studies. Int J Pharm. 2006;313:49-56.

10. Shen Q, Li W, Lin Y, et al. Modulating effect of polyethylene glycol on the intestinal transport and absorption of prednisolone, methylprednisolone and quinidine in rats by in-vitro and in-situ absorption studies. J Pharm Pharmacol. 2008;60:1633-1641.

11. Walle UK, Walle T. Taxol transport by human intestinal epithelial Caco-2 cells. Drug Metab Dispos. 1998;26:343-346.

12. Wang EJ, Casciano CN, Clement RP, Johnson WW. Active transport of fluorescent P-glycoprotein substrates: evaluation as markers and interaction with inhibitors. Biochem Biophys Res Commun. 2001;289: $580-585$.

13. Kim SW, Kwon HY, Chi DW, et al. Reversal of P-glycoproteinmediated multidrug resistance by ginsenoside Rg3. Biochem Pharmacol. 2003;65:75-82.

14. Yoshida N, Takagi A, Kitazawa H, Kawakami J, Adachi I. Inhibition of P-glycoprotein-mediated transport by extracts of and monoterpenoids contained in Zanthoxyli Fructus. Toxicol Appl Pharmacol. 2005;209: 167-173.

15. Yoshida N, Koizumi M, Adachi I, Kawakami J. Inhibition of P-glycoprotein-mediated transport by terpenoids contained in herbal medicines and natural products. Food Chem Toxicol. 2006;44:2033-2039.

16. Zhao K, Singh J. Mechanisms of percutaneous absorption of tamoxifen by terpenes: eugenol, d-limonene and menthone. J Control Release. 1998;55:253-260.

17. Sapra B, Jain S, Tiwary AK. Percutaneous permeation enhancement by terpenes: mechanistic view. AAPS J. 2008;10:120-132.

18. Young SC, Wang CJ, Hsu JD, Hsu JL, Chou FP. Increased sensitivity of Hep G2 cells toward the cytotoxicity of cisplatin by the treatment of piper betel leaf extract. Arch Toxicol. 2006;80:319-327.

19. Niel E, Scherrmann JM. Colchicine today. Joint Bone Spine. 2006;73: 672-678.

20. Grass GM, Sweetana SA. In vitro measurement of gastrointestinal tissue permeability using a new diffusion cell. Pharm Res. 1988; 5:372-376.

21. Saitoh H, Aungst BJ. Possible involvement of multiple P-glycoproteinmediated efflux systems in the transport of verapamil and other organic cations across rat intestine. Pharm Res. 1995;12:1304-1310.

22. Liang E, Chessic K, Yazdanian M. Evaluation of an accelerated Caco-2 cell permeability model. J Pharm Sci. 2000;89:336-345.
23. Eagling VA, Profit L, Back DJ. Inhibition of the CYP3A4-mediated metabolism and P-glycoprotein-mediated transport of the HIV-1 protease inhibitor saquinavir by grapefruit juice components. $\mathrm{Br} J \mathrm{Clin}$ Pharmacol. 1999;48:543-552.

24. Faassen F, Vogel G, Spanings H, Vromans H. Caco-2 permeability, P-glycoprotein transport ratios and brain penetration of heterocyclic drugs. Int J Pharm. 2003;263:113-122.

25. Zhang L, Zheng Y, Chow MS, Zuo Z. Investigation of intestinal absorption and disposition of green tea catechins by Caco-2 monolayer model. Int J Pharm. 2004;287:1-12.

26. Dahan A, Amidon GL. Grapefruit juice and its constituents augment colchicine intestinal absorption: potential hazardous interaction and the role of p-glycoprotein. Pharm Res. 2009;26:883-892.

27. Dahan A, Sabit H, Amidon GL. Multiple efflux pumps are involved in the transepithelial transport of colchicine: combined effect of p-glycoprotein and multidrug resistance-associated protein 2 leads to decreased intestinal absorption throughout the entire small intestine. Drug Metab Dispos. 2009;37:2028-2036.

28. Bittner B, Guenzi A, Fullhardt P, Zuercher G, González RC, Mountfield RJ. Improvement of the bioavailability of colchicine in rats by co-administration of D-alpha-tocopherol polyethylene glycol 1000 succinate and a polyethoxylated derivative of 12-hydroxy-stearic acid. Arzneimittelforschung. 2002;52:684-688.

29. Shaikh J, Ankola D, Beniwal V, Singha D, Kumar M. Nanoparticle encapsulation improves oral bioavailability of curcumin by at least 9-fold when compared to curcumin administered with piperine as absorption enhancer. European J Pharm Sci. 2009;37:223-230.

30. Italia J, Yahya M, Singh D, Kumar M. Biodegradable nanoparticles improve oral bioavailability of amphotericin b and show reduced nephrotoxicity compared to intravenous fungizone. Pharm Res. 2009; 26:1324-1331.

31. Singh A, Chaurasiya A, Jain G, Awasthi A, Asati D, Mishra G, Khar R, Mukherjee R. High performance liquid chromatography method for the pharmacokinetic study of bicalutamide SMEDDS and suspension formulations after oral administration to rats. Talanta. 2009;78: 1310-1314.

32. Shah NH, Carvajal MT, Patel CI, Infeld MH, Malick AW. Self-emulsifying drug delivery systems (SEDDS) with polyglycolyzed glycerides for improving in vitro dissolution and oral absorption of lipophilic drugs. Int J Pharm. 1994;106:15-23.

33. Constantinides PP. Lipid microemulsions for improving drug dissolution and oral absorption: physical and biopharmaceutical aspects. Pharm Res. 1995;2:1561-1572.

34. Kawakami K, Yoshikawa T, Hayashi T, Nishihara Y, Masuda K. Microemulsion formulation for enhanced absorption of poorly soluble drugs II. In vivo study. J Control Release. 2002;81:75-82.

35. Hirunpanich V, Sato H. Improvement of cyclosporine A bioavailability by incorporating ethyl docosahexaenoate in the microemulsion as an oil excipient. Eur J Pharm Biopharm. 2009;73:247-252.
International Journal of Nanomedicine

\section{Publish your work in this journal}

The International Journal of Nanomedicine is an international, peerreviewed journal focusing on the application of nanotechnology in diagnostics, therapeutics, and drug delivery systems throughout the biomedical field. This journal is indexed on PubMed Central, MedLine, CAS, SciSearch $®$, Current Contents $® /$ Clinical Medicine,

\section{Dovepress}

Journal Citation Reports/Science Edition, EMBase, Scopus and the Elsevier Bibliographic databases. The manuscript management system is completely online and includes a very quick and fair peer-review system, which is all easy to use. Visit http://www.dovepress.com/ testimonials.php to read real quotes from published authors. 